\title{
Aplicação de GPR para reconhecimento de possíveis zonas de contaminação em uma área de fossa séptica na Universidade Federal do Oeste do Pará., Santarém- PA.
}

Erverlon Bruno da Paixão Chaves, Universidade Federal do Oeste do Pará - UFOPA

Raimundo Nonato Colares Carneiro, Universidade Federal do Oeste do Pará - UFOPA

Anderson Conceição Mendes, Universidade Federal do Pará - UFPA

Marlon de Sousa Mota, Universidade Federal do Pará - UFPA

Copyright 2018, SBGf - Sociedade Brasileira de Geofísica

Este texto foi preparado para a apresentação no VIII Simpósio Brasileiro de Geofísica, Salinópolis, 18 a 20 de setembro de 2018. Seu conteúdo foi revisado pelo Comitê Técnico do VIII SimBGf, mas não necessariamente representa a opinião da SBGf ou de seus associados. É proibida a reprodução total ou parcial deste material para propósitos comerciais sem prévia autorização da SBGf.

\begin{abstract}
Studies using geophysics for scientific purposes are increasingly common. There are several methods for application, so GPR is a quick and simple method to use. From areas with signal attenuation, areas that may indicate contamination are known and delineated. In UFOPA this method was applied in a septic tank and was able to eliminate it. The sections $2 \mathrm{D}$ and $3 \mathrm{D}$ were created that allowed the occurrence of attenuated areas from $1 \mathrm{~m}$ deep to $3 \mathrm{~m}$, limited to the pit area. The attenuation was interpreted as percolation of effluents and showed that the structure of the fossa is not intact. These effluents can contaminate the soil as well as the free heat pump, thus rendering the well that supplies the entire university unusable.
\end{abstract}

\section{Introdução}

Nas últimas décadas, observa-se um elevado número de pesquisas que vem sendo desenvolvidas utilizando o método geofísico de GPR por sua facilitada aplicação e rápido resultado para a investigação de contaminação do subssolo e mais especialmente na detecção de plumas de contaminação (Ortega, 2006; Porsani et al. 2004; Mendes et al. 2017). Em áreas com ocupações urbanas, a falta de controle na construção de fossas e aterros, podem apresentar vazamento das redes coletoras de esgoto, são as principais fontes potenciais de contaminação das águas subterrâneas (Fetter, 2008). Uma das causas geradoras dos problemas está na infraestrutura inadequada das cidades, como redes de coleta de esgoto ineficientes ou inexistentes, ou a falta de planejamento dos centros urbanos, resultando em ocupações desordenadas que, muitas das vezes, estão em locais hidrogeologicamente mais vulneráveis à contaminação.

$\mathrm{Na}$ Universidade Federal do Oeste do Pará, em Santarém, existe uma fossa séptica que recebe todos os dejetos produzidos nesse campus. Distante aproximadamente $100 \mathrm{~m}$ dessa fossa está localizado o poço de água que abastece todo o campus (Fig. 1). Assim, esse trabalho teve como principal objetivo estudar a integridade dessa fossa, através de perfilagem com utilização de GPR em 2D e 3D para determinar se há vazamento e consequente contaminação do lençol freático.

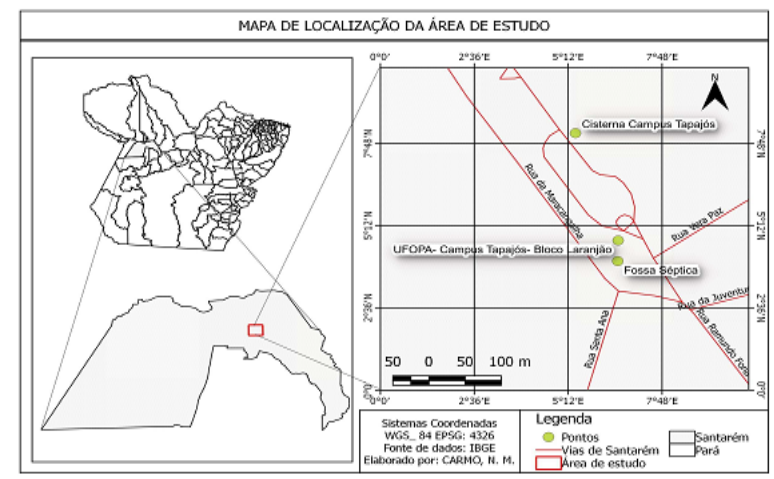

Figura 1. Mapa de localização da área de estudo com indicação dos objetos alvos.

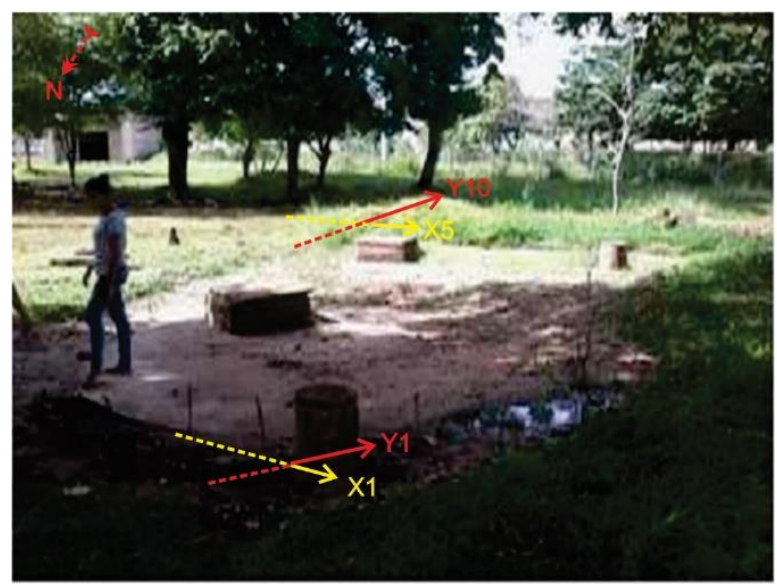

Figura 2. Fossa séptica nas áreas de Universidade Federal do Oeste do Pará.

\section{Metodologia}

Para determinar a integridade da fossa séptica, a área da mesma ( $5 \mathrm{~m} \times 10 \mathrm{~m})$ foi dividida em GRID's com linhas nos eixos $x$ e y espaçadas em $0,50 \mathrm{~m}$ resultado em uma área total de varredura em $10 \times 20 \mathrm{~m}$ (Fig. 2). A partir dessa varredura foram gerados 30 perfis $2 \mathrm{D}$ e, a partir deste foi gerado um perfil $3 \mathrm{D}$ da fossa com técnica de afastamento comum

O equipamento GRP utilizado foi o modelo GSSI 3000. Este equipamento consiste 6 itens; 2 antenas, 1 unidade transmissora, 1 unidade receptora, 1 unidade de controle e 1 microcomputador portátil. A frequência de operação 'e selecionada conectando as antenas ao equipamento. Neste trabalho foram utilizadas antenas de $270 \mathrm{MHz}$ que podem atingir até $6 \mathrm{~m}$ de profundidade a depender das propriedades físicas do meio.

$O$ processamento dos dados foi realizado com o programa ReflexW. Neste processamento levou-se em considerações as seguintes etapas: Correção estática 
$\rightarrow$ Remoção do ganho de cabeçalho $\rightarrow$ Ganho vertical $\rightarrow$ Remoção do background $\rightarrow$ Running average e Conversão do tempo em profundidade. As etapas deste trabalho estão indicadas na figura 3.

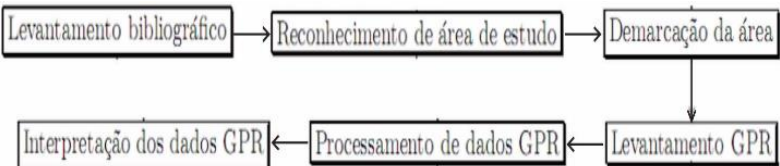
Figura 3. Métodos aplicados neste trabalho.

\section{Resultados}

Foram obtidas 30 linhas ao logo dos eixos $\mathrm{X}$ e $\mathrm{Y}$, separadas de 4 em 4 slices. Ao longo do eixo $X$, nas linhas 1 a 4 não se observa indícios de nenhuma contaminação abaixo da fossa séptica (Fig. 4). Nas linhas de 5 a 8 foi observado atenuação de sinal na profundidade de 1.40 metros, destacada pelo retângulo amarelo nas linhas 7 e 8 (Fig. 5) que podem indicar contaminação. Nas linhas 25 a 28 não se observa contaminação em nenhuma delas, porém são observadas as tubulações da fossa sobre a foram de hipérbole nas imagens (Fig. 6).

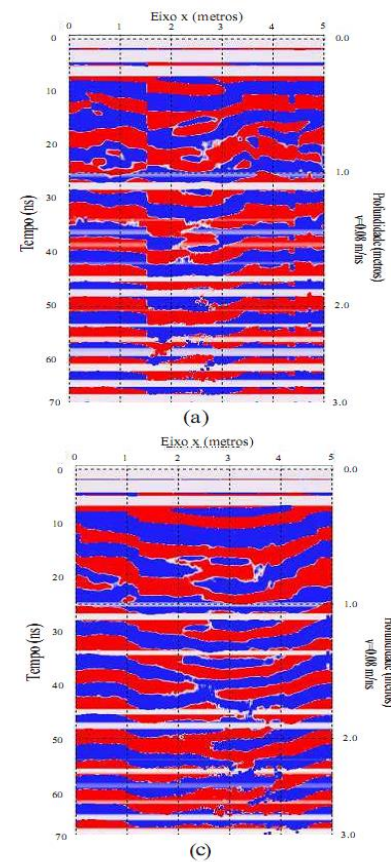

(c)

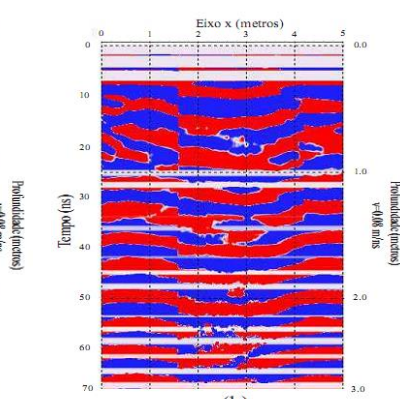

(b)

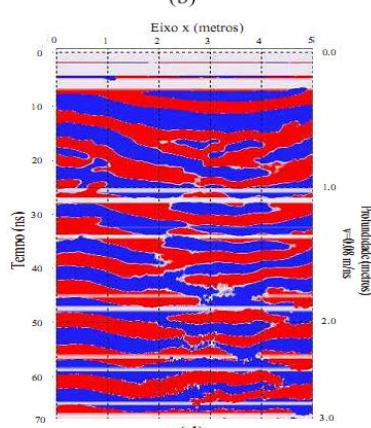

(d)
Figura 4: Linhas de 1 a 4, no eixo $X$. Não há contaminação detectada nestas linhas.

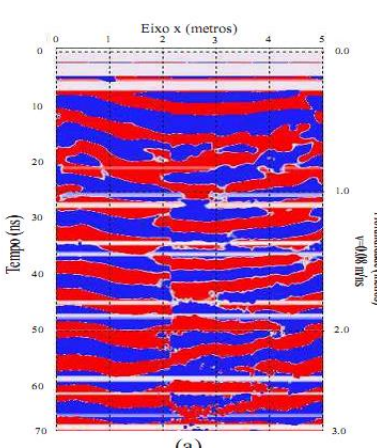

(a)
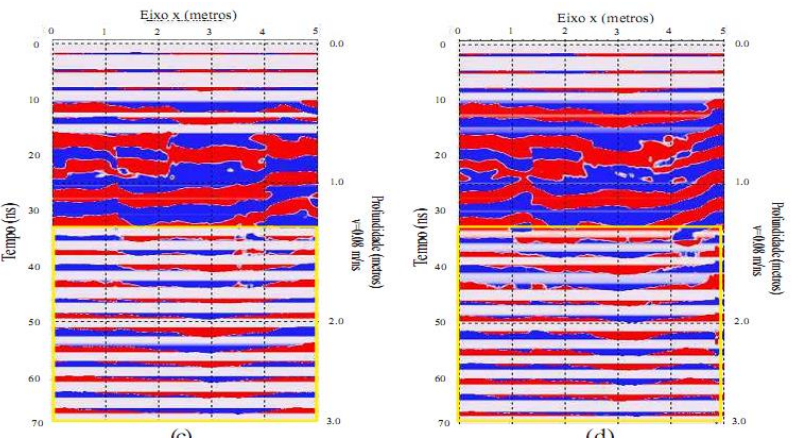

Figura 5. Linhas de 5 a 8, no eixo $X$, existe atenuação de sinal a partir de 1.40 metros de profundidade (retângulo amarelo) indicando possível contaminação.
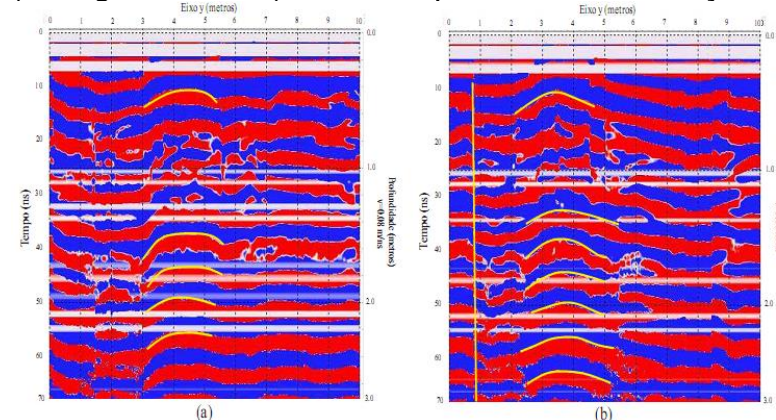

(a)

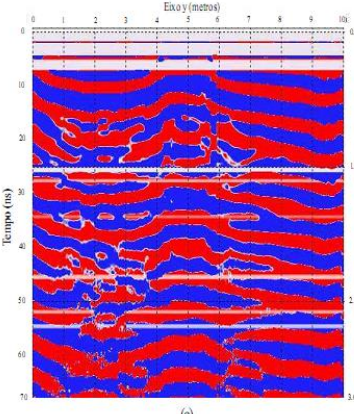

(b)

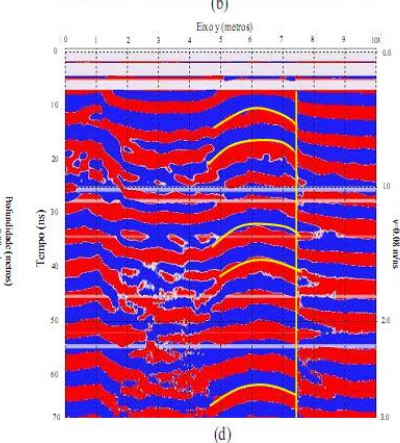

Figura 6: Linhas de 25 a 28, no eixo $X$ onde não há contaminação detectada nestas linhas. É possível observar a forma hiperbólica (zonas amarelas) que indica tubulações na área da fossa. 
Para visualizar de maneira mais clara a possível contaminação, observamos os resultados a partir do cubo 3D. Na porção frontal da fossa séptica é onde ocorre a maior atenuação do sinal podendo ser observado ao longo dos eixos $\mathrm{X}$ e $\mathrm{Y}$ (Fig. 7). Observase que a atenuação de sinal tem início a partir de 1.4 metros e atinge até 3 metros de profundidade. No eixo $Y$ essa atenuação é notada em dois intervalos, entre 3 e 5 metros e 8 e 10 metros.

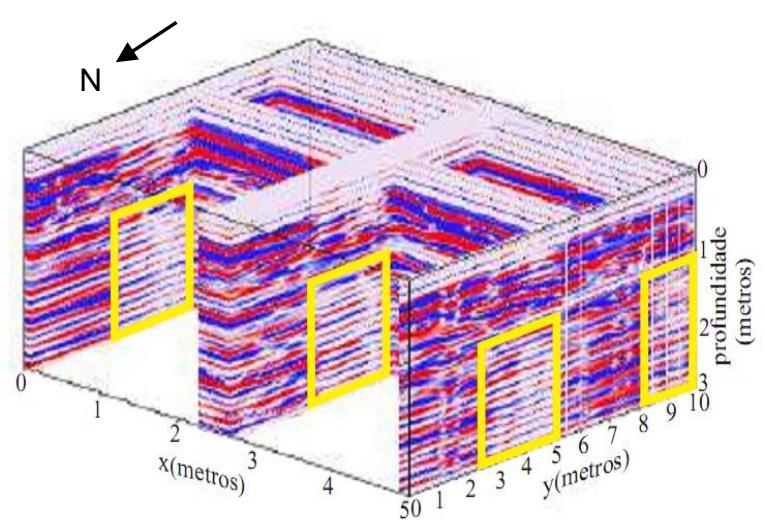

Figura 7: Cubo 3D da fossa séptica onde é possível observar as zonas com atenuação de sinal demarcadas em amarelo.

\section{Discussão e Conclusões}

Zonas com atenuação do sinal de GPR indicam alta condutividade e podem ser relacionadas com zonas contaminadas por degradação da matéria orgânica gerando chorume (Daniels et al. 1995; Atekwana et al. 2000). Os dados observados na fosse séptica da UFOPA demonstram que existe uma possível percolação de efluentes no solo e consequentemente contaminação no aquífero freático livre localizado abaixo da área da pesquisa. Essa contaminação é observada, principalmente, na parte frontal da fossa e indicada pela atenuação do sinal e mostra preferência de deslocamento em direção ao norte (Fig. 8).

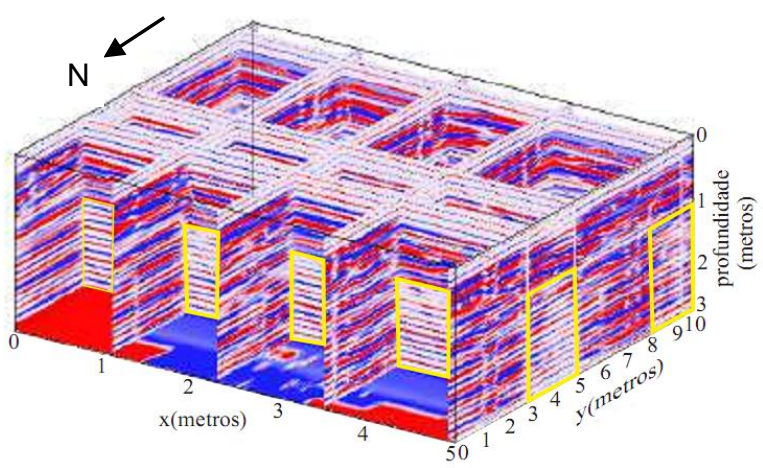

Figura 8: Cubo 3D da fossa séptica onde é possível observar as zonas com atenuação de sinal demarcadas em amarelo, com modificação da posição ao longo do eixo $X$ e $Y$
A percolação no solo ocorre em função da ausência de impermeabilização do substrato da fossa, e pode atingir a zona não saturada contaminando a zona freática livre. Como observado até o momento está pluma de contaminação está restrita a área delimitada da fossa (Fig. 8), entretanto caso não sejam tomadas medida, ela pode aumentar.

Caso essa contaminação atinja e contamine o lençol freático, a mesma, pode causar um problema maior ainda para a Universidade, pois pode ser necessário a inutilização do poço de água utilizado pela universidade. Esse fato ocorre, pois, o poço próximo a fossa. O substrato para construção do poço bem como da fossa é a Formação Alter do Chão sendo está com elevado conteúdo arenoso inconsolidado que facilita a percolação bem como o deslocamento do fluido.

Esse estudo é um passo inicial para e teve como principal objetivo testar a integridade da fossa séptica da UFOPA. Neles foi observado indícios que podem estar ocorrendo vazamento e consequente contaminação a partir da fossa séptica da UFOPA. Entretanto faz-se necessário a realização de outros levantamentos na área para melhor identificar a área de ocorrência da contaminação, bem como determinar a direção de migração da pluma de contaminação.

\section{Agradecimentos}

Os autores agradecem a Universidade Federal do Oeste do Pará - UFOPA pelo apoio logístico necessário para realização desta pesquisa.

\section{Referências}

Atekwana. E. A, Sauck, W. A, Werkema, D. D. (2000). Investigations of geoelectrical signatures at a hydrocarbon contaminated site. J. Appl.Geophys. 44:167-81.

Daniels, J. J, Roberts, R. L, Vendl, M. (1995). Ground penetrating radar for detection of liquid contaminants. J. Appl. Geophys 33 (1-3):195-207

Fetter, C. W (2008) Contaminant Hydrogeology. $2^{\text {nd }}$ Edition, Waveland Press Inc., Long Grove, 500 p. Journal of Applied Geophysics, 55, 199-209.

Mendes, A.C; Galvão, P; Souza, J. Silva, I \& Carneiro, N. (2017). Relations of the groundwater quality and disorderly occupation in an Amazon low-income neighborhood developed over a former dump area, Santarém/PA, Brazil. Environ Dev Sustain. DOI 10.1007/s10668-017-0040-8

Ortega, R. A. (2006). Localização e Delineamento de Pluma de Contaminação por Hidrocarbonetos através dos Métodos GPR e Tomografia Elétrica. Dissertação de mestrado. Instituto de Astronomia, Geofísica e Ciências Atmosféricas, Universidade de São Paulo. 115pag.

Porsani, J. L., Filho, W. M., Elis, V. R., Shimeles, F., Dourado, J. C., \& Moura, H. P. (2004). The use of GPR and VES in delineating a contamination plume in a landfill site: A case study in SE Brazil. Journal of Applied Geophysics, 55(3): 199-209. 